\title{
Optogalvanic Spectroscopy of Metastable States in $\mathrm{Yb}^{+}$
}

\author{
M.J. Petrasiunas - E.W. Streed - T.J. Weinhold • B.G. Norton • \\ D. Kielpinski
}

Received: date / Accepted: date

\begin{abstract}
The metastable ${ }^{2} \mathrm{~F}_{7 / 2}$ and ${ }^{2} \mathrm{D}_{3 / 2}$ states of $\mathrm{Yb}^{+}$are of interest for applications in metrology and quantum information and also act as dark states in laser cooling. These metastable states are commonly repumped to the ground state via the $638.6 \mathrm{~nm}{ }^{2} \mathrm{~F}_{7 / 2}$ $-{ }^{1} \mathrm{D}[5 / 2]_{5 / 2}$ and $935.2 \mathrm{~nm}{ }^{2} \mathrm{D}_{3 / 2}-{ }^{3} \mathrm{D}[3 / 2]_{1 / 2}$ transitions. We have performed optogalvanic spectroscopy of these transitions in $\mathrm{Yb}^{+}$ions generated in a discharge. We measure the pressure broadening coefficient for the $638.6 \mathrm{~nm}$ transition to be $70 \pm 10 \mathrm{MHz} \cdot \mathrm{mbar}^{-1}$. We place an upper bound of $375 \mathrm{MHz} /$ nucleon on the 638.6 $\mathrm{nm}$ isotope splitting and show that our observations are consistent with theory for the hyperfine splitting. Our measurements of the $935.2 \mathrm{~nm}$ transition extend those made by Sugiyama et al, showing well-resolved isotope and hyperfine splitting [1]. We obtain high signal to noise, sufficient for laser stabilisation applications [2].
\end{abstract}

Keywords ytterbium $\cdot$ ion $\cdot$ metastable $\cdot$ optogalvanic $\cdot$ spectroscopy

PACS $31.30 . \mathrm{Gs} \cdot 42.62 . \mathrm{Fi} \cdot 52.80 . \mathrm{Hc} \cdot 52.80 . \mathrm{Yr}$

M.J. Petrasiunas · E.W. Streed · B.G. Norton · D. Kielpinski Centre for Quantum Dynamics, Griffith University, Brisbane 4111, QLD, Australia

Tel.: +61-7-3735-3758

Fax: +61-7-3735-4426

E-mail: mattpetras@gmail.com

T.J. Weinhold

Centre for Quantum Computer Technology, Department of Physics, University of Queensland, Brisbane 4072, QLD, Australia

\section{Introduction}

The singly ionised ytterbium ( $\mathrm{Yb}$ ) atom has come under close interest for applications in a number of fields, including ion trap quantum computing and trapped ion optical frequency standards. Both of these areas provide motivation for investigation of the atomic structure of ytterbium ions. In this work, we study transitions from metastable states in $\mathrm{Yb}^{+}$that are important to both of these applications. Transitions in atoms with complex electronic structure, such as $\mathrm{Yb}^{+}$are a challenge to understand solely through theoretical calculations, thus necessitating experimental research to form an accurate picture of these electronic transitions.

The ${ }^{2} \mathrm{~S}_{1 / 2}-{ }^{2} \mathrm{~F}_{7 / 2}$ electric octupole transition in $\mathrm{Yb}^{+}$ is of particular interest for applications as an optical frequency standard $[3,4,5,6,7$. The excited state spontaneous emission lifetime of the transition is estimated to be on the order of 6 years $[6$. This remarkably long lifetime results in a natural transition linewidth on the order of $\mathrm{nHz}$ which provides an excellent basis for a highly accurate atomic clock transition. The clock laser excites ions into the long-lived ${ }^{2} \mathrm{~F}_{7 / 2}$ state, which is repumped to the ground state via the highly excited ${ }^{1} \mathrm{D}[5 / 2]_{5 / 2}$ state. Taylor et al. investigated the alternative $3.43 \mu \mathrm{m}$ ${ }^{2} \mathrm{~F}_{7 / 2}-{ }^{2} \mathrm{D}_{5 / 2}$ transition, finding it to be many times weaker than the $638.6 \mathrm{~nm}^{2} \mathrm{~F}_{7 / 2}-{ }^{1} \mathrm{D}[5 / 2]_{5 / 2}$ transition 8.

The principal laser cooling transition for $\mathrm{Yb}^{+}$in an ion trap is the $369.5 \mathrm{~nm}^{2} \mathrm{~S}_{1 / 2}-{ }^{2} \mathrm{P}_{1 / 2}$ transition. However the ${ }^{2} \mathrm{P}_{1 / 2}$ state has a branching ratio of $0.5 \%$ to decay into the metastable ${ }^{2} \mathrm{D}_{3 / 2}$ state [9], necessitating repumping via the $935.2 \mathrm{~nm}{ }^{2} \mathrm{D}_{3 / 2}-{ }^{3} \mathrm{D}[3 / 2]_{1 / 2}$ transition to return the ion to the ground state, as shown in Figure 1. In general, trapped ions that are repumped by $369.5 \mathrm{~nm}$ and $935.2 \mathrm{~nm}$ light will be kept within the 


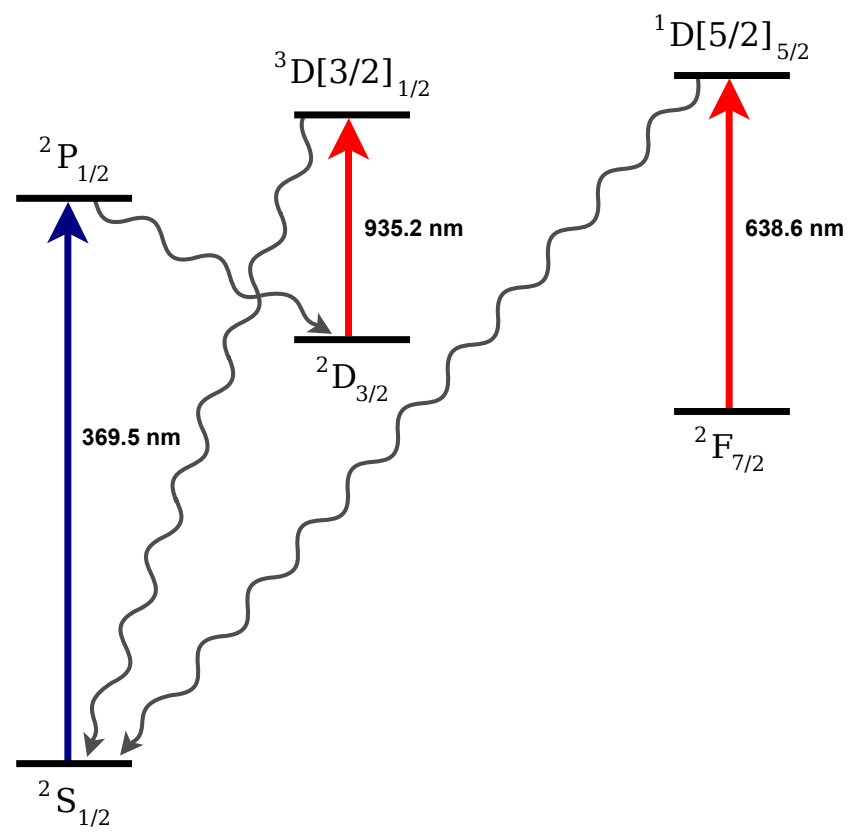

Fig. 1 Energy level diagram for $\mathrm{Yb}^{+}$. The $638.6 \mathrm{~nm}{ }^{2} \mathrm{~F}_{7 / 2}-$ ${ }^{1} \mathrm{D}[5 / 2]_{5 / 2}$ and $935.2 \mathrm{~nm}{ }^{2} \mathrm{D}_{3 / 2}-{ }^{3} \mathrm{D}[3 / 2]_{1 / 2}$ transitions studied in this work are shown in red. The $369.5 \mathrm{~nm}{ }^{2} \mathrm{~S}_{1 / 2}-{ }^{2} \mathrm{P}_{1 / 2}$ transition, used as principal laser cooling transition, has a $0.5 \%$ branching ratio to decay to the metastable ${ }^{2} \mathrm{D}_{3 / 2}$ state [9]. During trap operation, collisions with residual background gas may also cause an ion to reach the metastable ${ }^{2} \mathrm{~F}_{7 / 2}$ state [10]. Ions that fall into one of these 'dark' states must be repumped to the ground state via the $638.6 \mathrm{~nm}$ or the $935.2 \mathrm{~nm}$ transition.

cooling cycle. However, over long periods of operation collisions with residual background gas may also cause an ion to reach the metastable ${ }^{2} \mathrm{~F}_{7 / 2}$ state [10]. Ions in this state have fallen outside the range of the normal cooling cycle, and will remain in a 'dark' state until returned to the ${ }^{2} \mathrm{~S}_{1 / 2}$ state [11]. Despite the low rate of such occurrences, if the ions cannot be effectively isolated from collisions with an adequate vacuum pressure, then pumping of the ${ }^{2} \mathrm{~F}_{7 / 2}-{ }^{1} \mathrm{D}[5 / 2]_{5 / 2}$ transition must be performed in order to return ions that reach this dark state to the cooling cycle.

Due to the need for lasers to repump ions to the ground state from the metastable states discussed above, it is also important that these lasers can be stabilised to the transitions in question in order to counter the effects of frequency drift. We have previously demonstrated long-term absolute frequency stabilisation to ions in a discharge by employing the dichroic atomic vapor laser locking (DAVLL) method [2].

The frequency shift of the transition due to the hyperfine structure and isotope of $\mathrm{Yb}^{+}$is important when tuning or stabilising a laser to the transition. Also, due to the difficulty of theoretical predictions of such shifts on excited to excited state transitions such as this one, experimental determination of the shifts can provide a useful test of the theoretical methods that can be used to predict them. Thus it is necessary to perform spectroscopy in order to determine the relevant isotope shift and hyperfine structure constants.

Optogalvanic spectroscopy is an alternative method for investigating ion transitions in a discharge. The electrical properties of the plasma are dependent on the electronic state populations of atoms and ions in the plasma. When the plasma is illuminated by a laser resonant with a transition, the change in plasma resistance yields a measurable signal. This method allows for electrical detection of atomic transitions, resulting in far greater sensitivity than optical methods such absorption. This provides a particular advantage in studying transitions from metastable states such as we do in this paper, where the spectroscopic signal can be quite weak due to the limited population of these states. The necessary metastable states can be populated by collisions in the discharge [12,13.

In this paper we measure the optogalvanic spectra of the $638.6 \mathrm{~nm}^{2} \mathrm{~F}_{7 / 2}-{ }^{1} \mathrm{D}[5 / 2]_{5 / 2}$ and $935.2 \mathrm{~nm}{ }^{2} \mathrm{D}_{3 / 2}-$ ${ }^{3} \mathrm{D}[3 / 2]_{1 / 2}$ transitions. We also provide a bound on isotope splitting for the $638.6 \mathrm{~nm}$ transition and show that the hyperfine constant is consistent with theory. We investigate the broadening processes present in both sets of spectra, eliminating pressure broadening of the 638.6 $\mathrm{nm}$ line in order to improve our measurement. In the case of the $935.2 \mathrm{~nm}$ transition, we obtain well-resolved isotopic and hyperfine lines, extending the measurements made by Sugiyama et al [1].

\section{Experimental Method}

We performed optogalvanic spectroscopy in $\mathrm{Yb}$ hollowcathode lamps containing Ne buffer gas at pressures of 6.7 mbar and 0.67 mbar. All hollow-cathode discharges used had the same geometry. The 6.7 mbar discharge was generated in a sealed Hamamatsu L2783-70NE-YB hollow cathode lamp, and was used to perform spectroscopy on both metastable transitions of interest.

A second purpose-built discharge was prepared inside a vacuum chamber, intended to allow for variation in the buffer gas pressure within the discharge with the purpose of operation at 0.67 mbar. This was done in order to see an expected $10 \times$ reduction in pressure broadening. The chamber was evacuated directly by a rotary vacuum pump and vacuum pressure was monitored by a thermocouple gauge, sensitive down to $10^{-3}$ mbar. After evacuation, the chamber was baked at $180^{\circ} \mathrm{C}$ over a period of $2-3$ days. At this point, a base pressure of 0.013 mbar was attained. A noble buffer gas was then able to be introduced via a needle valve. The buffer gas pressure was monitored with a thermocouple gauge. 


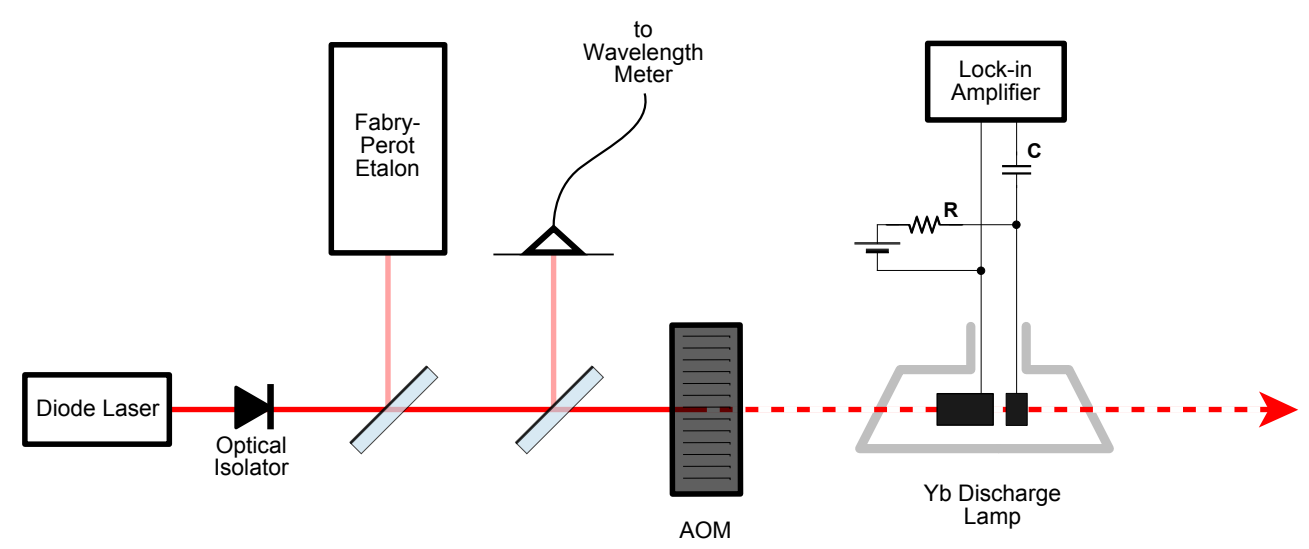

Fig. 2 Schematic of setup used for optogalvanic spectroscopy. Laser diodes tuned by current and temperature to be on resonance with $638.6 \mathrm{~nm}$ and $935.2 \mathrm{~nm}$ transitions, with fine and course frequency reference from etalon and wavelength meter respectively. Optical isolation is present immediately after the laser. The laser beam is amplitude modulated by an acousto-optic modulator (AOM). Both 6.7 mbar and 0.67 mbar discharges were used. The signal due to laser excitation is filtered and demodulated by the lock-in amplifier.

Sanyo DL5038-021 laser diodes were tuned for use at $638.6 \mathrm{~nm}$ using temperature and operating current. Behaviour close to the desired wavelength varied largely from diode to diode. The diodes were operated without an external cavity, running single-frequency with a linewidth less than $30 \mathrm{MHz}$, where the output was optically isolated to prevent optical feedback and damage to the diode. A Fabry-Perot etalon with an FSR of $300 \mathrm{MHz}$ was used as a relative frequency reference to measure the frequency splittings and peak widths of the measured spectrum. Absolute wavelength measurement for tuning purposes was performed using a HighFinesse WS5-332 Wavemeter with $\pm 0.001 \mathrm{~nm}$ accuracy. The implementation of the setup for optogalvanic spectroscopy followed the schematic shown in Figure 2 .

The $935.2 \mathrm{~nm}$ spectroscopy setup was similar to that for the $638.6 \mathrm{~nm}$ line. A single mode tuneable external cavity laser was constructed with an Axcell Photonics laser diode (M9-935-0200-S30) and a $1200 \mathrm{~g} / \mathrm{mm}$ holographic grating. The frequency was continuously tuneable over a range of $13.7 \mathrm{GHz}$ by tuning the external cavity length with a piezo-electric actuator and synchronously varying the laser diode current. The laser spectral mode quality was monitored with a confocal Fabry-Perot spectrometer with a free spectral range of $478 \pm 8 \mathrm{MHz}$ and a finesse of 11 . The observed spectrum bounds the $935.2 \mathrm{~nm}$ laser linewidth to below $50 \mathrm{MHz}$, though linewidths of a few $\mathrm{MHz}$ are typical for this laser configuration. The absolute wavelength was measured with a Burleigh WA-1100 Wavemeter (accuracy $\pm 0.001 \mathrm{~nm}$ ). The $935.2 \mathrm{~nm}$ light was fibre coupled for delivery to the sealed discharge lamp. This provided 19 $\mathrm{mW}$ of $935.2 \mathrm{~nm}$ power at the discharge lamp in a laser beam spot with a diameter of $1.70 \pm 0.02 \mathrm{~mm}\left(1 / \mathrm{e}^{2}\right)$, corresponding to a maximum intensity of $1.674 \pm 0.039$ $\mathrm{W} \cdot \mathrm{cm}^{-2}$.

In order for the optogalvanic signal to be distinguished from the electronic background noise, the optical beam was modulated at a set reference frequency using either amplitude or frequency modulation. We demodulated the signal using lock-in detection. A significant advantage of this method is that it allows for removal of $1 / f$ noise, which would otherwise dominate the signal, allowing a high signal-to-noise ratio to be obtained. In these experiments we chopped the beam with an acousto-optic modulator (AOM), switching the beam intensity between zero and maximum intensity.

\section{Results for $638.6 \mathrm{~nm}{ }^{2} F_{7 / 2}-{ }^{1} \mathrm{D}[5 / 2]_{5 / 2}$ transition}

We obtained optogalvanic spectra from discharge lamps operating with a discharge current of $3 \mathrm{~mA}$ at $6.7 \mathrm{mbar}$ and $2 \mathrm{~mA}$ at $0.67 \mathrm{mbar}$, for which the maximum signal amplitude was obtained. The $638.6 \mathrm{~nm}$ laser power used at the lamp was $10-13 \mathrm{~mW}$, chopped at a modulation frequency of $10 \mathrm{kHz}$. The signals for both the sealed 6.7 mbar discharge and the low-pressure 0.67 mbar discharge with Ne buffer gas are shown in Figure 3 .

The linewidth of the signal obtained at a buffer gas pressure of 6.7 mbar was found to be approximately $1.9 \mathrm{GHz}$ (FWHM). The observed linewidth decreases to approximately $1.5 \mathrm{GHz}$ as the pressure is reduced to 0.67 mbar. There is some structure in the low-pressure peak that could be attributed to the splitting of the 


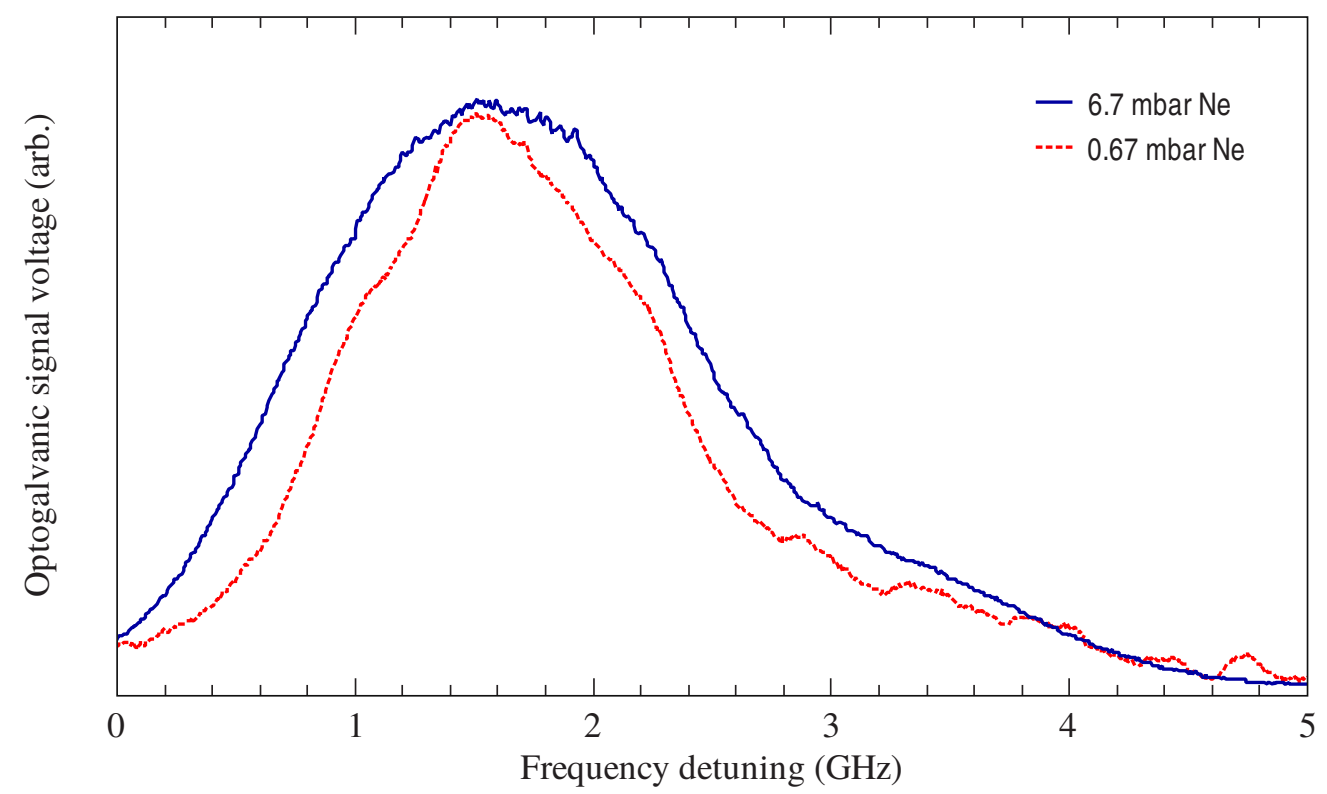

Fig. 3 Optogalvanic spectra of the $638.6 \mathrm{~nm}^{2} \mathrm{~F}_{7 / 2}-{ }^{1} \mathrm{D}[5 / 2]_{5 / 2}$ transition line in $\mathrm{Yb}^{+}$, as observed in $\mathrm{Yb}^{+}$discharges with 6.7 mbar (blue line) and 0.67 mbar (red line) of Ne buffer gas. The spectra are normalised for ease of comparison. The reduction in signal linewidth from $1.9 \mathrm{GHz}$ at 6.7 mbar to $1.5 \mathrm{GHz}$ at 0.67 mbar is attributed to pressure broadening.

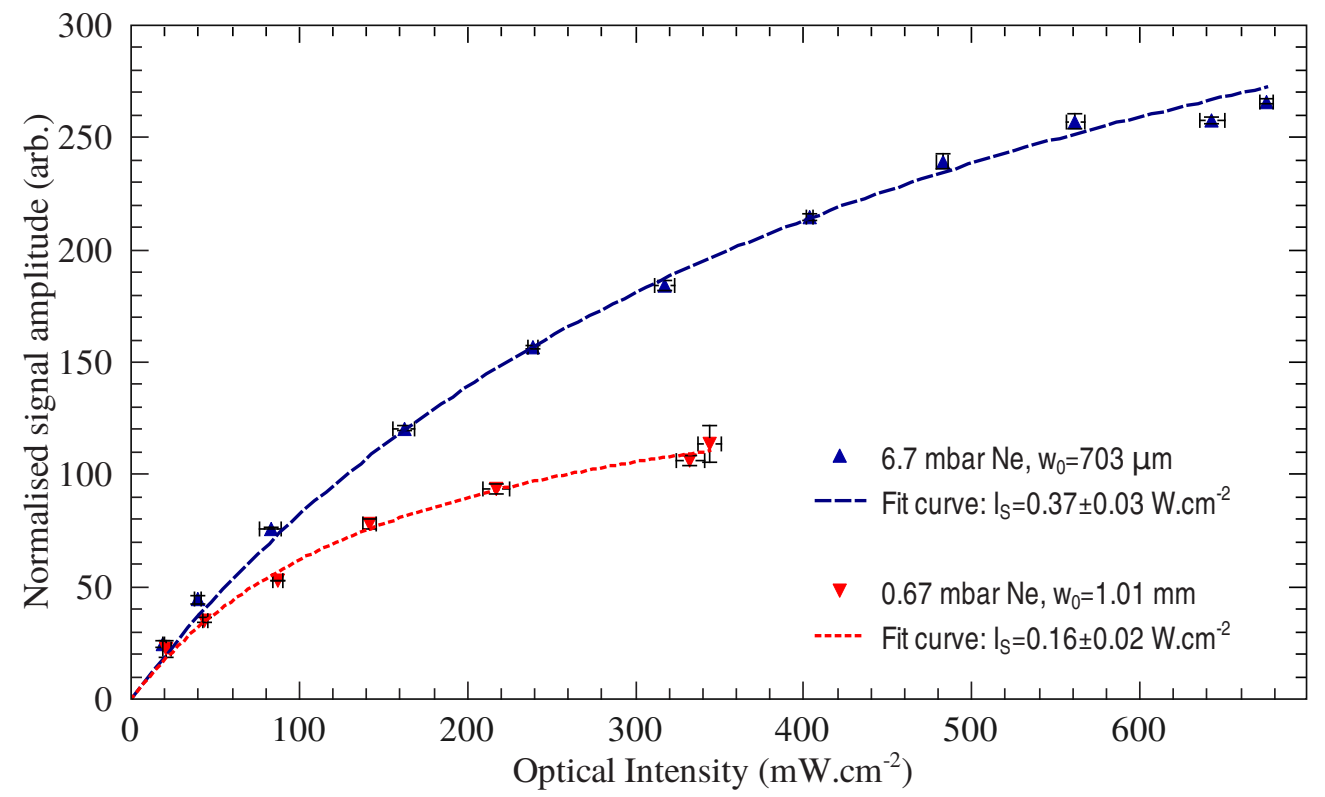

Fig. 4 The relationship of optogalvanic signal amplitude to optical intensity for both pressure levels. Blue colouring and upward triangles denote the measurements taken at 6.7 mbar Ne buffer gas pressure, whilst red and downward triangles denote the measurements taken at 0.67 mbar. The broken lines denote the fitted saturation curves. For ease of comparison, the signals are normalised to have equal slope near zero optical power. 
$638.6 \mathrm{~nm}$ line. The frequency axis of the plots is determined by taking the frequency spacing of Fabry-Perot transmission peaks to be equal to the Fabry-Perot FSR.

The optogalvanic signal amplitude was measured for a range of optical powers producing the saturation curve shown in Figure 4. A pure pressure broadening model predicts a signal at maximum amplitude of the form

$V_{s i g} \propto \frac{s}{1+s}$

to describe the effect of saturation on the optogalvanic signal, where the saturation parameter $s=\frac{I}{I_{S}}$ is the ratio of optical intensity to the saturation value and $V_{\text {sig }}$ is the signal amplitude [14. The saturation intensity $I_{S}$ is dependent on the homogeneous linewidth $\Gamma_{H}$ as shown by

$I_{S}=\frac{2 \pi^{2} h c}{3 \lambda^{3}} \Gamma_{H}$

where the homogeneous linewidth varies linearly with the pressure $P$ according to the Stern-Vollmer equation $\Gamma_{H}=\Gamma_{0}+\alpha P$ for constants $\Gamma_{0}$ and $\alpha$ [15].

Figure 4 shows experimental data on the saturation of optogalvanic response. The signals were fitted with Equation (1) and the slopes near zero intensity have been normalised for ease of comparison. The measured optical power is combined with the beam waist size $w_{0}$ inside the discharge to give the optical intensity, and hence allow calculation of the saturation intensity $I_{S}$ for each measured curve.

A beam profiling camera was used to find the $1 / e^{2}$ beam radius $w_{0}$ inside the 6.7 mbar discharge to be $730 \mu \mathrm{m}$, resulting in an estimated saturation intensity of $0.37 \pm 0.03 \mathrm{~W} \cdot \mathrm{cm}^{-2}$. The $0.67 \mathrm{mbar}$ discharge had a saturation intensity of $0.16 \pm 0.02 \mathrm{~W} \cdot \mathrm{cm}^{-2}$ from the fitted saturation curve, where the measured beam waist $w_{0}$ was $1.01 \mathrm{~mm}$. As the effects of inhomogeneous broadening on the saturation behaviour cannot be ruled out, these values for the saturation intensity are upper bounds.

Using Equation 22 and the calculated saturation intensities gives maximum homogeneous linewidth estimates of $0.74 \pm 0.06 \mathrm{GHz}$ for the 6.7 mbar discharge, and $0.32 \pm 0.04 \mathrm{GHz}$ for the measurements of the $0.67 \mathrm{mbar}$ discharge. The difference between the linewidths in the high- and low-pressure discharges is $0.42 \pm 0.08 \mathrm{GHz}$, which agrees with the difference in total linewidths measured from the spectra. This gives values for the $\Gamma_{0}$ and $\alpha$ coefficients, such that $\Gamma_{0}=0.27 \pm 0.07 \mathrm{GHz}$ and $\alpha=70 \pm 10 \mathrm{MHz} \cdot \mathrm{mbar}^{-1}$. The value for $\Gamma_{0}$ is the remaining broadening not induced by pressure, whilst $\alpha$ is a property of the discharge and buffer gas used.
The vacuum system used to construct the lowpressure discharge allowed for use of different buffer gases in place of Ne. Ar buffer gas was shown to generate $\mathrm{Yb}^{+}$ions, using a UV spectrometer to observe fluorescence from the $369.5 \mathrm{~nm}$ line to confirm this for our apparatus. However whilst Martensson-Pendrill et al. demonstrated optogalvanic spectroscopy of the 369.5 nm ${ }^{2} \mathrm{~S}_{1 / 2}-{ }^{2} \mathrm{P}_{1 / 2}$ line using Ar buffer gas [16], we were unable to observe an optogalvanic signal from the 638.6 $\mathrm{nm}$ transition. No such issue occurred with a Ne buffer gas, allowing for both $\mathrm{Yb}^{+}$generation and the expected optogalvanic response. The strong $\mathrm{Ne}^{*}$ transition line at $638.47 \mathrm{~nm}$ near the $638.6 \mathrm{~nm} \mathrm{Yb}^{+}$transition did not appear to compromise the measurement. We observed a DC offset in the optogalvanic response at the 638.6 $\mathrm{nm}$ line, attributed to the wings of the extremely strong $\mathrm{Ne}^{*}$ line.

\section{Results for $935.2 \mathrm{~nm}{ }^{2} \mathrm{D}_{3 / 2}-{ }^{3} \mathrm{D}[3 / 2]_{1 / 2}$ transition}

An optogalvanic spectrum with resolved isotope splittings was measured for the ${ }^{2} \mathrm{D}_{3 / 2}-{ }^{3} \mathrm{D}[3 / 2]_{1 / 2}$ transition at $935.2 \mathrm{~nm}$ in the sealed discharge lamp. Overlapping spectra were taken to measure the response over the $25.4 \mathrm{GHz}$ span from 935.159 to $925.233 \mathrm{~nm}$. Figure 5 shows a $10 \mathrm{GHz}$ span encompassing the observed peaks, taken with $18 \mathrm{~mW}$ of $935.2 \mathrm{~nm}$ laser power and amplitude modulated at $20 \mathrm{kHz}$. In these measurements, the optogalvanic signal increased monotonically with current, so the maximum rated lamp current of $10 \mathrm{~mA}$ was used. The four predominant peaks occurred at wavelengths of 935.173, 935.180, 935.187, and 935.197 nm. No peaks were observed outside of the region shown in figure 5 and the spectra is in qualitative agreement with previously published work [1]. The hyperfine structure of $\mathrm{Yb}^{+} 171$ and 173 can be seen to overlap with the peaks from 174,172, and 170. From measurements in trapped ions [17] the $\mathrm{Yb}^{+} 172$ transition is known to be only $-110 \mathrm{MHz}$ from the $F=1-F^{\prime}=0$ transition of $\mathrm{Yb}^{+} 171$. Combined with the known hyperfine splitting of $\mathrm{Yb}^{+} 171$ allows the assignment of these features. A FWHM of $535 \pm 7 \mathrm{MHz}$ was measured on the 176 peak and is representative of the single isotope broadening. This peak is free from distortions induced by overlap between peaks due to the hyperfine splittings in $\mathrm{Yb}^{+} 171$ and 173 . The integrated optogalvanic signal over the $\mathrm{Yb}^{+} 176$ peak is $12 \pm 1 \%$ of the total integrated signal, consistent with the natural abundance of $12.7 \%$. Other peak assignments were verified through a combination of known wavelengths and hyperfine splittings from trapped ion experiments and 


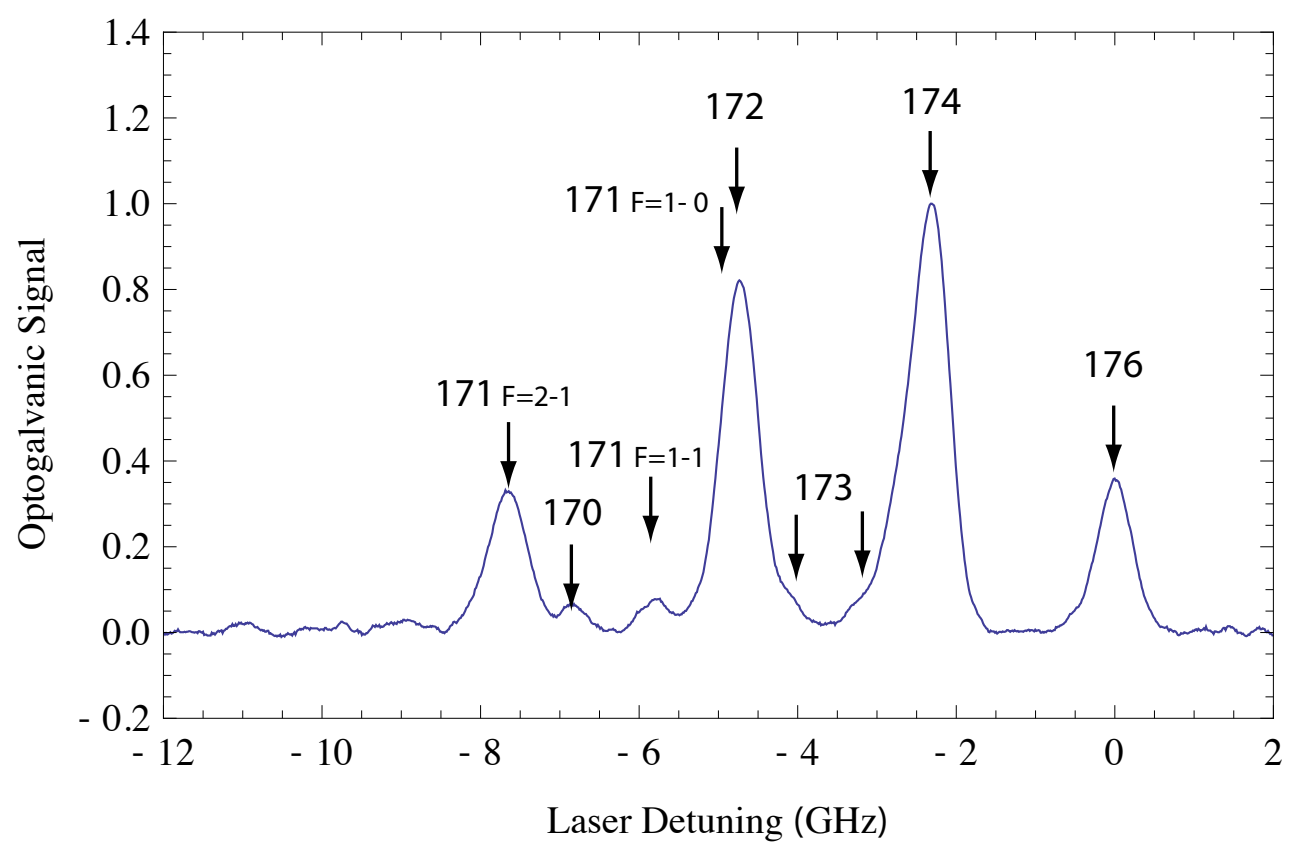

Fig. 5 Optogalvanic spectrum of the ${ }^{2} \mathrm{D}_{3 / 2}-{ }^{3} \mathrm{D}[3 / 2]_{1 / 2}$ transition in $\mathrm{Yb}^{+}$at 935.2 nm in a sealed discharge lamp. Laser detuning is with respect to the $\mathrm{Yb}^{+} 176$ peak. The discharge lamp was run at its maximum rated current of $10 \mathrm{~mA}$. The observed 176 $\mathrm{Yb}^{+}$peak FWHM linewidth is $535 \pm 7 \mathrm{MHz}$. The laser illuminated the lamp with $19 \mathrm{~mW}$ of light and a maximum intensity of $1.674 \pm 0.039 \mathrm{~W} \cdot \mathrm{cm}^{-2}$. Locations for $\mathrm{Yb}^{+} 173$ peaks are estimates from the total integrated signal weighting and the predicted location of the transition centroid from the isotopic shift trend.

weighting based on natural abundance and ClebschGordon coefficients. We measure a splitting between the $174 / 172$ peaks of $2.39 \pm 0.01 \mathrm{GHz}$ and a splitting of $2.33 \pm 0.01 \mathrm{GHz}$ between the $174 / 176$ peaks. This gives an average isotope shift of $1.18 \pm 0.03 \mathrm{GHz} /$ nucleon. The optogalvanic signal showed a linear dependence in laser power for $2.5 \mathrm{~mW}$ to $18 \mathrm{~mW}$ with no evidence of saturation. Changing the lamp current from $3 \mathrm{~mA}$ to $14 \mathrm{~mA}$ also produced a linear increase in signal. Similar optogalvanic spectra were observed with a blind hollow cathode lamp (Cathodeon 3UX Yb, Ne buffer gas). With a lock-in amplifier time constant of $\tau=300$ ms the maximum signal to noise observed was 58 .

\section{Discussion}

Hyperfine and isotope splitting have strong effects on the optogalvanic lineshape. In the case of the $935.2 \mathrm{~nm}$ transition, the splitting due to these effects is well resolved. Previous spectroscopic work on this transition also gives a good idea of the composition of the expected spectrum [1].

In the case of the $638.6 \mathrm{~nm}$ transition, the broadening of the transition linewidth due to homogeneous and inhomogeneous sources makes resolution of the splitting difficult. Using theoretical estimates, the splitting spectrum that we expect to observe for the $638.6 \mathrm{~nm}$ in the well-resolved limit would contain 5 strong lines. This is based on the natural isotopic abundance of $\mathrm{Yb}^{+}$ and the fact that the large number of hyperfine levels for $\mathrm{Yb}^{+} 173(I=5 / 2)$ will be far weaker in intensity than the transition lines for the $171,172,174$ and 176 isotopes [18. We theoretically estimate the hyperfine splitting to be several $\mathrm{GHz}$ for $\mathrm{Yb}^{+} 171$. The hyperfine splitting of the $\mathrm{Yb}^{+}$isotopes with even mass numbers will be zero, as these isotopes carry no nuclear spin.

Although broadening of the linewidth prevents clear resolution of hyperfine and isotope structure, deviation from the Gaussian shape on the higher-frequency side of the spectrum at the higher pressure of 6.7 mbar shows discernible structure in the observed peak. Hints of further structure become evident in the main peak at the lower pressure of 0.67 mbar, suggesting that this main peak is made up of several broadened lines.

As stated in Section 3, we obtained a $0.42 \pm 0.08 \mathrm{GHz}$ difference between the maximum homogeneous linewidths in the 6.7 mbar and 0.67 mbar discharges from measurements of signal saturation. This agrees with the 0.4 $\mathrm{GHz}$ difference in total linewidth measured from the optogalvanic spectra, showing that the absolute difference is dominantly due to pressure broadening. However, as the achieved reduction in linewidth is significantly less than the expected factor of $10 \times$ for pressure broadening, there is evidently another source of broadening. 
The residual pressure broadening at 0.67 mbar is $<50$ $\mathrm{MHz}$, given that the expected linear relationship holds.

The contribution of power broadening on the linewidth of the optogalvanic transition can be estimated to be $<100 \mathrm{MHz}$, by estimating a natural linewidth on the order of $10 \mathrm{MHz}$. The lack of any significant contribution by power broadening was also confirmed by illuminating the discharge with powers of less than $1 \mathrm{~mW}$ and finding no noticeable difference in linewidth.

From the analysis above, it is evident that Doppler broadening is the only possible remaining broadening mechanism for the low-pressure measurements of the $638.6 \mathrm{~nm}$ line. The residual linewidth of the $638.6 \mathrm{~nm}$ transition appears to arise from the overlap of multiple unresolved Doppler-broadened lines. The temperature associated with a given Doppler linewidth is given by

$T=\frac{M \lambda^{2} \Gamma_{D}^{2}}{8 k_{B} \ln 2}$

for atoms of mass $M$ and a Doppler-broadened FWHM linewidth $\Gamma_{D}$ [15]. Results in the literature suggest that an expected temperature for a hollow-cathode discharge is $300 \mathrm{~K}$, which would equate to a Doppler linewidth of approximately $440 \mathrm{MHz}$ at $638.6 \mathrm{~nm} \mathrm{[12}$. This level of Doppler broadening corresponds well with the optogalvanic spectrum in Figure 3 observed at 0.67 mbar, being at a level where multiple lines spaced by $<0.5$ $\mathrm{GHz}$ are not be fully resolved. This lends weight to the possibility of structure observed in the low-pressure optogalvanic spectrum.

Preliminary calculations of hyperfine structure were performed by Dr. W. M. Itano of the National Institute of Standards and Technology, Boulder, CO, USA. The hyperfine $A$ and $B$ constants of the ${ }^{1} \mathrm{D}[5 / 2]_{5 / 2}$ state and the ${ }^{2} \mathrm{~F}_{7 / 2}$ state have been estimated by a multiconfiguration Dirac-Hartree-Fock method, utilising the GRASP package of atomic structure programs [19,20]. The method is similar to the cross-optimisation method used to calculate the hyperfine structure of the $\mathrm{Hg}^{+}$ion [21, in which orbitals are optimised, stepwise, on different configurations.

At each step, the previously optimised orbitals are held fixed. In the first step, the $\{1 s, 2 s, 2 p, 3 s, 3 p, 3 d, 4 s$, $4 p, 4 d, 5 s, 5 p\}$ core orbitals and $\{4 f, 6 s\}$ valence orbitals are variationally optimised on the $J=(5 / 2,7 / 2)$ levels of the $4 f^{13} 6 s^{2}$ configuration. Next, keeping the previously optimised orbitals fixed, the $\{5 f, 7 s\}$ orbitals are determined by minimising the energy of the ground state, which is $4 f^{14} 6 s$ in a first approximation. The $5 d$ orbital is optimised on the $4 f^{14} 5 d(J=3 / 2,5 / 2)$ levels. The $6 p$ orbital is optimised on the $4 f^{14} 6 p(J=$ $1 / 2,3 / 2)$ levels. In the final step, the $\{8 s, 7 p, 6 d, 6 f\}$ orbitals are optimised on the lowest-energy odd-parity $(J=9 / 2,11 / 2,13 / 2)$ levels.
This set of orbitals is used to generate a set of basis states by making single and double replacements from a set of reference configurations. The final approximate wavefunctions are calculated by relativistic configuration-interaction, i.e. by diagonalising the DiracCoulomb Hamiltonian matrix in this basis. The hyperfine constants are evaluated by evaluating the expectation values of the hyperfine energy operators in these wavefunctions.

For the ${ }^{1} \mathrm{D}[5 / 2]_{5 / 2}$ state, $A(\mathrm{Yb} 171)=199 \mathrm{MHz}$, $A(\mathrm{Yb} 173)=-55 \mathrm{MHz}$, and $B(\mathrm{Yb} 173)=-1720 \mathrm{MHz}$. For the ${ }^{2} \mathrm{~F}_{7 / 2}$ state, $A(\mathrm{Yb} 171)=1105 \mathrm{MHz}$, $A(\mathrm{Yb} 173)=-304 \mathrm{MHz}$, and $B(\mathrm{Yb} \mathrm{173)}=-3680$ $\mathrm{MHz}$. The only one of these constants for which there is an experimental measurement is $A(\mathrm{Yb} 171)=905.0(0.5)$ $\mathrm{MHz}$ [8], measured for the ${ }^{2} \mathrm{~F}_{7 / 2}$ state, which is about $20 \%$ lower than the calculated value. Our data is consistent with the splitting for the ${ }^{1} \mathrm{D}[5 / 2]_{5 / 2}$ state being much smaller than the ${ }^{2} \mathrm{~F}_{7 / 2}$ state splitting, agreeing with Dr. Itano's theoretical estimate.

No theoretical results are available for the isotope shift of the $638.6 \mathrm{~nm}$ transition, but the normal mass shift is known to be small (on the order of a few $\mathrm{MHz}$ ). The specific mass shift and field shift however are known to be far larger, providing the dominant contribution to the isotope shift. We can put an upper limit of approximately $375 \mathrm{MHz} /$ nucleon on the isotope shift, given that there are at least three equally-spaced strong lines in the main peak with a combined linewidth of $1.5 \mathrm{GHz}$. The poorly resolved features in the low-pressure spectrum have approximately a $750 \mathrm{MHz}$ spacing and could correspond to the isotopes 172,174 and 176 of $\mathrm{Yb}^{+}$.

The interpretation of the $935.2 \mathrm{~nm}$ transition optogalvanic spectra is substantially less complicated than that for $638.6 \mathrm{~nm}$ transition. The transition wavelengths for all isotopes excepting 168 (abundance 0.13\%) and 173 have previously been measured in trapped ion experiments. Our estimate of the isotope shift for the ${ }^{2} \mathrm{D}_{3 / 2}-{ }^{3} \mathrm{D}[3 / 2]_{1 / 2}$ transition is in agreement with the $1.3 \pm 0.1 \mathrm{GHz} /$ nucleon from previous discharge lamp work [1. The small discrepancy between our measurement of $1.18 \pm 0.03 \mathrm{GHz} /$ nucleon and the $1.42 \pm 0.07$ $\mathrm{GHz} /$ nucleon from measurements in single trapped ions [17. is most likely due to shifts induced in the lamp measurements by overlap of the 170, 172 and 174 isotope peaks with the hyperfine structure of $\mathrm{Yb}^{+} 171$ and 173. Agreement between the optogalvanic peaks and the published transition wavelengths for specific isotopes allows us to bound the overall lamp pressure shift as $< \pm 60 \mathrm{MHz} \cdot \mathrm{mbar}^{-1}$, limited by the absolute accuracy of our wavemeter. We also place an upper limit of $80 \pm 1 \mathrm{MHz} \cdot \mathrm{mbar}^{-1}$ on the pressure broadening coefficient $\alpha$ for this transition, assuming that the linewidth 
of the $\mathrm{Yb}^{+} 176$ line is entirely due to pressure broadening. The prospects for long term frequency stabilisation to the $935.2 \mathrm{~nm}$ optogalvanic signal are encouraging given the observed signal to noise ratio of 58 for subsecond averaging.

\section{Conclusion}

We have measured the optogalvanic response of the $638.6 \mathrm{~nm}{ }^{2} \mathrm{~F}_{7 / 2}-{ }^{1} \mathrm{D}[5 / 2]_{5 / 2}$ and $935.2 \mathrm{~nm}{ }^{2} \mathrm{D}_{3 / 2}-$ ${ }^{3} \mathrm{D}[3 / 2]_{1 / 2}$ transitions in singly ionised $\mathrm{Yb}^{+}$. We observe linewidths of $1.9 \mathrm{GHz}$ and $1.5 \mathrm{GHz}$ for the 638.6 $\mathrm{nm}$ optogalvanic peak, using discharges with 6.7 mbar and 0.67 mbar of Ne buffer gas, respectively, where Doppler broadening is expected to be the cause of these large linewidths and the inability to resolve the splittings. We performed measurements of the saturation curves in both pressure regimes, calculating the pressure broadening coefficient to be $70 \pm 10 \mathrm{MHz} \cdot \mathrm{mbar}^{-1}$, and confirming that the source of the remaining broadening is inhomogeneous. The broadening prevents full resolution of transition lines in the main spectral peak, however our data is consistent with a much larger splitting of the ${ }^{2} \mathrm{~F}_{7 / 2}$ state than the ${ }^{1} \mathrm{D}[5 / 2]_{5 / 2}$ state, as predicted by our theoretical estimate. We can provide an upper limit to the isotope splitting of approximately $375 \mathrm{MHz} /$ nucleon. In our measurements of the $935.2 \mathrm{~nm}$ optogalvanic spectrum, we resolve with high signal-tonoise the peaks of the dominant isotopes of $\mathrm{Yb}^{+}$. We provide an upper bound of $80 \pm 1 \mathrm{MHz} \cdot \mathrm{mbar}^{-1}$ for the pressure broadening coefficient of this transition. The optogalvanic spectra observed in the 6.7 mbar discharge expand on the measurement made by Sugiyama et al [1]. Our results demonstrate that a high signal to noise ratio can be achieved within a discharge for measurements of both transitions, also showing that both transitions are well suited for applications in laser stabilisation [2.

Acknowledgements We thank Dr. Wayne Itano for providing calculations of the $638.6 \mathrm{~nm}$ transition hyperfine structure. This work was supported by the U.S. Air Force Office of Scientific Research under AOARD Contracts FA2386-09-1-4132 and FA238610-1-4090 and by the Australian Research Council (ARC) under DP0773354 (Kielpinski), DP0877936 (Streed, Australian Postdoctoral Fellowship) and Howard Wiseman's Federation Fellowship FF0458313.

\section{References}

1. K. Sugiyama, J. Yoda, IEEE Trans. Instrum. Meas. 44, 140 (1995)

2. E.W. Streed, T.J. Weinhold, D. Kielpinski, Appl. Phys. Lett. 93, $071103(2008)$
3. M. Roberts, P. Taylor, G.P. Barwood, P. Gill, H.A. Klein, W.R.C. Rowley, Phys. Rev. Lett. 78, 1876 (1997)

4. S.A. Webster, P. Taylor, M. Roberts, G.P. Barwood, P. Gill, Phys. Rev. A 65, 052501 (2002)

5. P.J. Blythe, S.A. Webster, H.S. Margolis, S.N. Lea, G. Huang, S.-K. Choi, W.R.C. Rowley, P. Gill, R.S. Windeler, Phys. Rev. A 67, 020501 (2003)

6. K. Hosaka, S.A. Webster, P.J. Blythe, A. Stannard, D. Beaton, H.S. Margolis, S.N. Lea, P. Gill, IEEE Trans. Instrum. Meas. 54, 759 (2005)

7. K. Hosaka, S.A. Webster, A. Stannard, B.R. Walton, H.S. Margolis, P. Gill, Phys. Rev. A 79, 033403 (2009)

8. P. Taylor, M. Roberts, G.M. Macfarlane, G.P. Barwood, W.R.C. Rowley, P. Gill, Phys. Rev. A 60, 2829 (1999)

9. N. Yu, L. Maleki, Phys. Rev. A 61, 022507 (2000)

10. S. Olmschenk, K.C. Younge, D.L. Moehring, D.N. Matsukevich, P. Maunz, C. Monroe, Phys. Rev. A 76, 052314 (2007)

11. H. Lehmitz, J. Hattendorf-Ledwoch, R. Blatt, H. Harde, Phys. Rev. Lett. 62, 2108 (1989)

12. P. Hannaford, Contemp. Phys. 24, 251 (1983)

13. B. Barbieri, N. Beverini, A. Sasso, Rev. Mod. Phys. 62, 603 (1990)

14. H.J. Metcalf, P. Van der Straten, Laser Cooling and Trapping (Springer Verlag, New York, 1999)

15. A.E. Siegman, Lasers (University Science Books, Sausalito, California, 1986)

16. A. Martensson-Pendrill, D.S. Gough, P. Hannaford, Phys. Rev. A 49, 3351 (1994)

17. J.J. McLoughlin, A.H. Nizamani, J.D. Siverns, R.C. Sterling, M.D. Hughes, B. Lekitsch, B. Stein, S. Weidt, W.K. Hensinger, Phys. Rev. A 83, 013406 (1994)

18. R.J. Hayden, D.C. Hess, M.G. Inghram, Phys. Rev. 75, 322 (1949)

19. F.A. Parpia, C. Froese Fischer, I.P. Grant, Comput. Phys. Commun. 94, 249 (1996)

20. P. Jönsson, F.A. Parpia, C. Froese Fischer, Comput. Phys. Commun. 96, 301 (1996)

21. T. Brage, C. Proffitt, D.S. Leckrone, Astrophysical Journal 513, 524 (1999) 